\title{
Management of isolated oligohydramnios in Korea: a questionnaire-based study of clinical practice patterns among the members of the Korean Society of Maternal Fetal Medicine
}

\author{
Seung Woo Yang, MD, PhD ${ }^{1}$, Yihua Jin, MD, MS ${ }^{1}$, Eun bi Jang, MD ${ }^{1}$, Hee Sun Kim, MD, PhD², \\ In Sook Sohn, MD, PhD ${ }^{1}$, Han Sung Kwon, MD, PhD ${ }^{1}$, Han-Sung Hwang, MD, PhD ${ }^{1}$

\begin{abstract}
Department of Obstetrics and Gynecology, ${ }^{1}$ Research Institute of Medical Science, Konkuk University School of Medicine, Seoul; ${ }^{2}$ Ilsan Paik Hospital,
\end{abstract} \\ Inje University College of Medicine, Goyang, Korea
}

\section{Objective}

The aim of this survey was to investigate the recommendations and clinical practice patterns of the Korean Society of Maternal and Fetal Medicine (KSMFM) members, regarding management of isolated oligohydramnios (IO).

\section{Methods}

From December 2018 to February 2019, questionnaires were e-mailed to the KSMFM members at 257 institutes that are listed by the Korean Statistical Information Services (KOSIS) as suitable labor premises. Responses to the seven questions on the management of $\mathrm{IO}$, from diagnosis to treatment, were evaluated.

\section{Results}

A total of 72 KSMFM members responded to this survey. Nearly all participants (90.1\%) used the amniotic fluid index (AFI) as the primary method for estimating amniotic fluid volume. The majority of the participants (73.6\%) believed that IO was a risk factor for adverse pregnancy outcomes, including abnormal fetal heart rate $(73.6 \%)$, need for cesarean delivery (58.3\%), intrauterine fetal demise (52.8\%), and meconium aspiration syndrome (50\%). Almost $70 \%$ of the participants believed that induction of labor might decrease perinatal morbidities, and that late-preterm to early-term period (36-38 gestational weeks) was a suitable timeframe for delivery, if the fetus was sufficiently grown and antenatal testing revealed reassuring results. Less than half of the participants $(47.2 \%)$ believed that maternal oral or intravenous hydration was a useful intervention for IO management.

\section{Conclusions}

KSMFM members preferred labor induction at late-preterm to early-term, to decrease perinatal morbidity in cases of IO, although it was still uncertain whether labor induction improved the outcomes. Further prospective studies are needed regarding 10 management.

Keywords: Oligohydramnios; Questionnaire; Clinical practice pattern

\section{Introduction}

Amniotic fluid protects the fetus, reflects the fetal condition, and provides an environment for various fetal movements, including breathing $[1,2]$. Oligohydramnios is associated with maternal and fetal hypertension, diabetes, congenital anomalies, fetal growth restriction, and preterm rupture of membranes. Independently, it has been reported to reflect
Received: 2020.03.08. Revised: 2020.04.24. Accepted: 2020.05.05. Corresponding author: Han-Sung Hwang, MD, PhD

Department of Obstetrics and Gynecology, Konkuk University School of Medicine, 120 Neungdong-ro, Gwangjin-gu, Seoul 05029, Korea E-mail: hwanghs@kuh.ac.kr

https://orcid.org/0000-0003-0622-0701

Articles published in Obstet Gynecol Sci are open-access, distributed under the terms of the Creative Commons Attribution Non-Commercial License (http://creativecommons. org/licenses/by-nc/3.0/) which permits unrestricted non-commercial use, distribution, and reproduction in any medium, provided the original work is properly cited.

Copyright $\odot 2020$ Korean Society of Obstetrics and Gynecology 


\section{Obstetrics \& Gynecology Science}

Seung Woo Yang, et al. Management of isolated oligohydramnios

uteroplacental insufficiency and chronic fetal hypoxia $[3,4]$. When oligohydramnios is diagnosed by chance during the routine second and third trimester pregnancy examination, clinicians tend to recommend a variety of tests to identify its causes, such as the presence of congenital malformations and the size of the fetus, and to conduct fetal surveillance including Doppler studies.

Oligohydramnios without any evidence of either maternal or fetal abnormalities is known as "isolated" oligohydramnios (IO) [5]. In low-risk pregnancies, the incidence of 10 has been estimated as 1.5\% [6]. IO management guidelines are not well defined as its effect on perinatal outcomes remains controversial. However, a recent meta-analysis has suggested that 10 in low-risk pregnancy was associated with adverse outcomes such as neonatal intensive care unit (NICU) admission, cesarean delivery, and meconium aspiration syndrome [7]. A systematic review and meta-analysis suggested that oral or intravenous maternal hydration improves amniotic fluid volume; however, further evaluation of its clinical application is required [8].

Given the potentially severe complications and lack of clear management guidelines, the diagnosis of IO can affect pregnancy management and the decision to induce labor. In fact, a 2009 survey of Society for Maternal Fetal Medicine (SMFM) has revealed that approximately $80 \%$ of the participants declared either that induction of labor in cases of $10 \mathrm{did}$ not reduce perinatal morbidity or that they were uncertain whether it did. However, despite being unsure of its benefits, $96 \%$ of the practitioners leaned towards intervention [9].

In the absence of 10 management guidelines, insights into obstetricians' clinical practice patterns are required. This survey aimed to investigate the knowledge and clinical practice patterns associated with 10 management among the members of the Korean Society of Maternal Fetal Medicine (KSMFM).

\section{Materials and methods}

From December 2018 to February 2019, questionnaires were sent to 272 labor units listed by the Korean Statistical Information Services (KOSIS) as suitable labor premises. The institutes included 41 obstetrics departments within university hospitals, 86 obstetrics departments within general hospitals, and 145 private obstetric hospitals. The survey comprised of seven questions and was distributed by e-mail.

IO was defined as an ultrasonography-based diagnosis of abnormally low amniotic fluid with intact membranes in the absence of a fetal growth restriction, fetal anomalies, or significant maternal comorbidity. The participants were asked to report on their management of such cases, based on their personal and their unit's standard protocols. The questionnaire was composed of three parts: 1) measurement and related perinatal outcomes of 10,2 ) preferences for induction of labor in presence of 10 atthird trimester and 3) preferences of oral or intravenous hydration and intraamniotic fluid infusion in IO. In this questionnaire-based survey, respondents were asked to provide details of their individual characteristics, such as medical practice setting, age, sex, and the year of their certification as obstetrical specialists. The questionnaire was adapted from an earlier survey for SMFM members regarding the same matter [9].

Table 1. Characteristics of obstetricians who responded to the survey, regarding their clinical practice patterns in oligohydramnios management $(n=72)$

\begin{tabular}{|c|c|c|}
\hline Characteristics & & Values \\
\hline \multirow[t]{2}{*}{ Sex } & Male & $33(45.8)$ \\
\hline & Female & $39(54.2)$ \\
\hline \multirow[t]{5}{*}{ Age (yr) } & & $45.6 \pm 6.8$ \\
\hline & $30-39$ & $15(20.8)$ \\
\hline & $40-49$ & $37(51.4)$ \\
\hline & $50-59$ & $18(25.0)$ \\
\hline & $\geq 60$ & $2(2.8)$ \\
\hline \multirow[t]{4}{*}{ Type of labor unit } & OB/GYN clinic & $3(4.2)$ \\
\hline & OB/GYN hospital ${ }^{a)}$ & $16(22.2)$ \\
\hline & $\begin{array}{l}\text { OB/GYN department at } \\
\text { a general hospital }\end{array}$ & $7(9.7)$ \\
\hline & $\begin{array}{l}\text { OB/GYN department at } \\
\text { a university hospital }\end{array}$ & $46(63.9)$ \\
\hline Duration of experience (yr) & & $15.2 \pm 7.4$ \\
\hline \multirow{5}{*}{$\begin{array}{l}\text { Average number of monthly } \\
\text { hospital-based deliveries }\end{array}$} & $\leq 50$ & $27(37.5)$ \\
\hline & $51-100$ & $20(27.8)$ \\
\hline & $101-150$ & $14(19.5)$ \\
\hline & $151-200$ & $6(8.3)$ \\
\hline & $\geq 201$ & $5(6.9)$ \\
\hline
\end{tabular}

Data presented as mean \pm standard deviation or number (\%). $\mathrm{OB} / \mathrm{GYN}$, obstetrics and gynecology.

a) With more than one staff obstetrician. 


\section{Obstetrics \& Gynecology Science}

Vol. 63, No. 5, 2020

\section{Results}

\section{Participants' characteristics}

A total of $72 \mathrm{KSMFM}$ members responded to this survey. The mean age of the respondents was $45.6 \pm 6.8$ years. Among the sample, $45.8 \%$ and $54.2 \%$ respondents were males and females, respectively. The average duration of clinical obstetric experience was $15.2 \pm 7.4$ years. About two-thirds of the participants (45/72) were working at hospitals with $>50$ deliveries each month (Table 1).

\section{Management patterns of isolated oligohydramnios cases}

Questionnaires and responses are summarized in Table 2. Most participants used the amniotic fluid index (AFI) as the primary method for estimating amniotic fluid volume (Question 1). The majority of participants (73.6\%) declared that they believed 10 was a risk factor for significant adverse pregnancy outcomes (Question 3). Moreover, over 50\% of the participants believed that abnormal fetal heart rate $(73.6 \%)$, cesarean delivery (58.3\%), intrauterine fetal demise (52.8\%), and meconium aspiration syndrome (50.0\%) were adverse outcomes associated with IO. Similarly, approximately $70 \%$ of the participants considered 10 to indicate a need for delivery induction in the third trimester (Question 4). Over $50 \%$ of the participants thought early-term (37-38 gestational weeks) delivery was appropriate in cases of 10 , when the fetus was sufficiently grown and the results of antenatal testing were reassuring (Question 5). Less than half of the participants $(34 / 72,47.2 \%)$ believed that oral or intravenous hydration was a useful intervention in patients with IO. Among these, $50.0 \%$ (17/34) selected oral hydration for a short-term effect (Question 6). The majority of participants $(63 / 72,87.5 \%)$ did not perform amniotic infusion in pregnancies with 10 (Question 7).

\section{Discussion}

Amniotic fluid physiologic dynamics during pregnancy are complex. Abnormal volumes of amniotic fluid are associated with various adverse pregnancy outcomes [10]. Oligohydramnios is estimated to occur in $0.5-5.5 \%$ of all pregnancies, depending on the method of amniotic fluid volume measurement [11-13]. Oligohydramnios without any evidence of anatomical, functional, or chromosomal abnormalities is called "isolated" oligohydramnios. Since IO indicates a lowrisk pregnancy and the oligohydramnios measurements have a low accuracy, the 10 management protocols are debatable $[5,14]$.

In the present questionnaire-based survey, the majority of respondents used AFI to measure amniotic fluid volume. The gold standard for the amniotic fluid volume estimation is the dye dilution test [15]. However, this test is invasive, and is rarely required or used. Thus, single deepest pocket (SDP), measuring $\leq 2 \mathrm{~cm}$, or $A F I \leq 5 \mathrm{~cm}$ are the commonly used indirect criteria for confirmation of oligohydramnios [16]. However, SDP and AFI often grossly under- or over-estimate oligohydramnios $[17,18]$. Although, neither of the two assessments have been proved to be superior to the other; when the AFI was used, significantly more cases of oligohydramnios were diagnosed, and more women underwent interventions [19].

Patients with oligohydramnios have high-risk pregnancies, associated with adverse outcomes such as admission to the NICU, meconium stained amniotic fluid, increased rate of cesarean section, low Apgar score at birth, fetus that is small for gestational age, low umbilical cord $\mathrm{pH}$, and respiratory distress syndrome. However, since 10 indicates a low-risk pregnancy, its evaluation as a risk factor for adverse pregnancy outcomes is still controversial [20]. In a recent meta-analysis of 12 studies, Shrem et al. [10] reported that 10 at term was associated with significantly higher rates of labor induction, cesarean section, and short-term neonatal morbidity. Moreover, Rabie et al. [7] reported that 10 in low-risk pregnancy increased the risk of NICU admission, cesarean delivery for fetal distress, and meconium aspiration syndrome. In the present survey, over $50 \%$ of the participants reported cesarean delivery, abnormal fetal heart rate, and meconium aspiration syndrome in neonates as adverse outcomes associated with 10. This was in accordance with the previous SMFM survey (Fig. 1). However, the proportion of the response of "no significant attributable risks" differed in the two surveys. This may be attributed to the increased understanding of 10 in perinatal outcomes during the last 10 years.

Due to the associated adverse outcomes, interventions, such as labor induction, are important considerations when treating patients with confirmed 10 in the third trimester of pregnancy. In 2009, the SMFM questionnaire-based survey revealed that approximately $80 \%$ of the participants believed 


\section{Obstetrics \& Gynecology Science}

Seung Woo Yang, et al. Management of isolated oligohydramnios

Table 2. Isolated oligohydramnios survey and responses

\begin{tabular}{|c|c|}
\hline Questionnaire item & No. (\%) \\
\hline 1. Which methods do you currently use most frequently to evaluate the amniotic fluid volume? & $72(100.0)$ \\
\hline Amniotic fluid index (AFI) & $65(90.1)$ \\
\hline Maximal vertical pocket & $7(9.9)$ \\
\hline Subjective assessment & 0 \\
\hline 2. In your opinion, is isolated oligohydramnios a risk factor for any of the following outcomes: (Check all that apply) & $72(100.0)$ \\
\hline Cesarean delivery & $42(58.3)$ \\
\hline Abnormal fetal heart rate & $53(73.6)$ \\
\hline Intrauterine fetal demise & $38(52.8)$ \\
\hline Neonatal acidosis & $15(20.8)$ \\
\hline Meconium aspiration syndrome & $36(50)$ \\
\hline Low Apgar score & $33(45.8)$ \\
\hline Low birth weight & $21(29.2)$ \\
\hline NICU admission & $34(47.2)$ \\
\hline Neonatal brain injury & $8(11.1)$ \\
\hline No significant attributable risk & $1(1.4)$ \\
\hline $\begin{array}{l}\text { 3. Does induction of labor in cases of isolated oligohydramnios at term decrease perinatal morbidity associated with the } \\
\text { above-listed outcomes? }\end{array}$ & $72(100.0)$ \\
\hline Yes & $53(73.6)$ \\
\hline No & $15(20.8)$ \\
\hline Unknown & $4(5.6)$ \\
\hline 4. Is a case of isolated oligohydramnios in third trimester an indication for labor induction? & $72(100)$ \\
\hline Yes & $50(69.4)$ \\
\hline No & $22(30.6)$ \\
\hline $\begin{array}{l}\text { 5. For a sufficiently grown fetus with isolated oligohydramnios and reassuring antenatal testing results, what is the } \\
\text { maximum gestational age before recommending inducing labor? }\end{array}$ & $72(100.0)$ \\
\hline 34 weeks & $5(6.9)$ \\
\hline 35 weeks & $3(4.2)$ \\
\hline 36 weeks & $8(11.1)$ \\
\hline 37 weeks & $26(36.1)$ \\
\hline 38 weeks & $15(20.8)$ \\
\hline 39 weeks & $7(9.7)$ \\
\hline 40 weeks & $7(9.7)$ \\
\hline No significant risk attributable to gestational age & $1(1.4)$ \\
\hline $\begin{array}{l}\text { 6. Should oral or intravenous hydration be used to gain additional amniotic fluid before intervening in a patient with } \\
\text { isolated oligohydramnios? }\end{array}$ & $72(100.0)$ \\
\hline Yes & $34(47.2)$ \\
\hline Oral hydration for short-term effect & $17(23.6)$ \\
\hline Intravenous hydration for short-term effect & $8(11.1)$ \\
\hline Oral hydration for long-term effect & $8(11.1)$ \\
\hline Intravenous hydration for long-term effect & $1(1.4)$ \\
\hline No & $38(52.8)$ \\
\hline 7. In your practice, is amniotic infusion used in patients with isolated oligohydramnios? & $72(100.0)$ \\
\hline Yes & $9(12.5)$ \\
\hline No & $63(87.5)$ \\
\hline
\end{tabular}




\section{Obstetrics \& Gynecology Science}

Vol. 63, No. 5, 2020

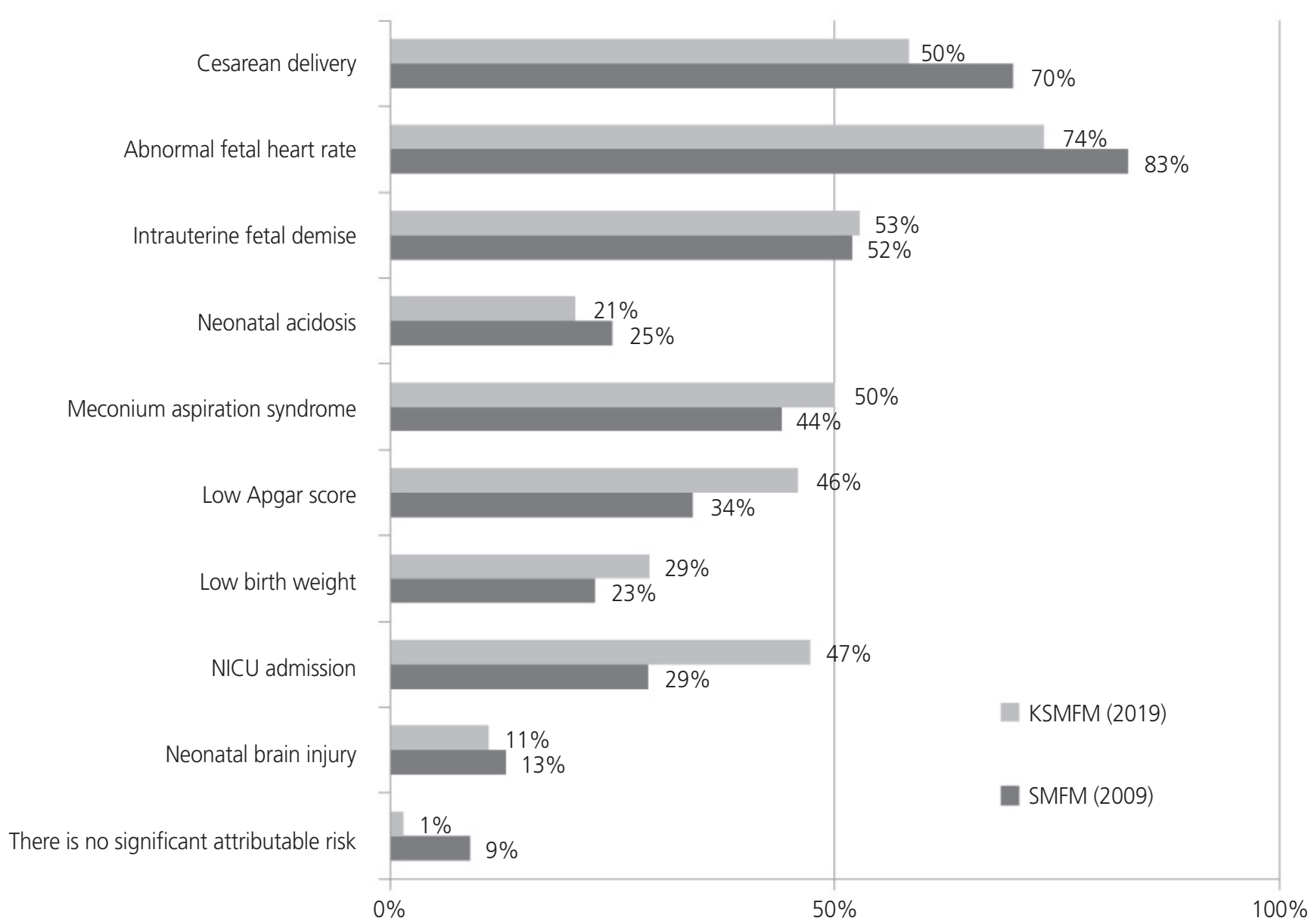

Fig. 1. Comparison of Society for Maternal Fetal Medicine (SMFM; 2009) and Korean Society of Maternal Fetal Medicine (KSMFM; 2019) survey regarding the relation between perinatal outcomes and isolated oligohydramnios. NICU, neonatal intensive care unit.

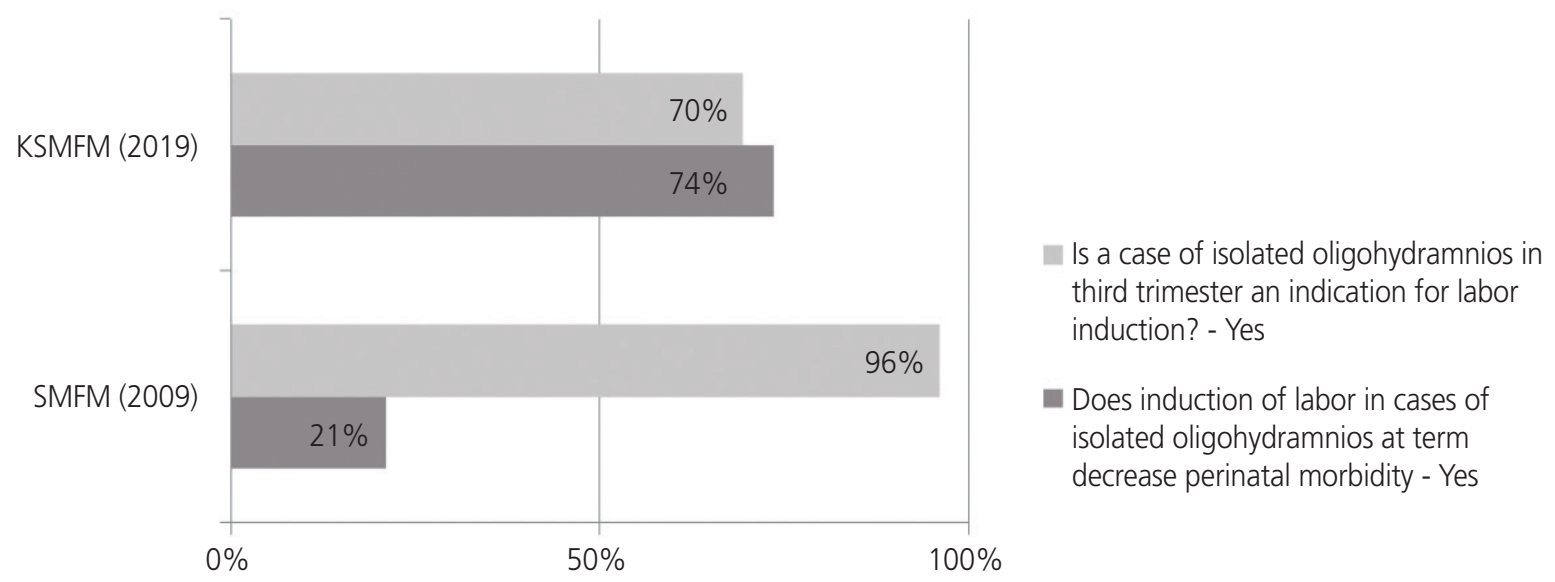

Fig. 2. Comparison of Society for Maternal Fetal Medicine (SMFM; 2009) and Korean Society of Maternal Fetal Medicine (KSMFM; 2019) survey regarding labor induction in isolated oligohydramnios. 


\section{Obstetrics \& Gynecology Science}

Seung Woo Yang, et al. Management of isolated oligohydramnios

that induction of labor in cases of IO did not reduce perinatal morbidity or that they were uncertain whether it did. However, despite being unsure of its benefits, $96 \%$ of the practitioners recommended this intervention [9]. Conversely, in the present survey, nearly $74 \%$ of the participants believed that induction of labor reduced perinatal morbidity and almost $70 \%$ of them indicated considering induction of labor in the third trimester of pregnancy with IO (Fig. 2). These results show that the perceptional uncertainty of labor induction for IO has reduced in the last 10 years. Furthermore, $68 \%$ of the participants declared that late pre-term to early-term period (36-38 gestational weeks) was a suitable timeframe for delivery. In a recent study, Rabies et al. have reported that the lack of required data precludes any conclusions regarding optimal timeframe for delivery in pregnancies with 10 [7]. Conversely, Brzezinski-Sinai et al. [14] have reported that in cases of $\mathrm{IO}$ in late pre-term, induction of labor may reduce the risk of perinatal morbidities despite a premature birth. The American College of Obstetricians and Gynecologists (ACOG) recommendations state that 36.0-37.7 weeks of gestation are a suitable timeframe for delivery in cases with 10 [21]. Given these considerations, until 35.6 weeks of gestation, conservative management is required. Further, until delivery, intensive fetal surveillances including fetal biophysical profile and Doppler analysis to evaluate placental dysfunction are needed $[22,23]$. In the KSMFM survey of 70 members in 2013, Oh reported that fetal surveillances were performed twice a week $(20 \%)$, every week $(43 \%)$ or every other week (35\%), and $46 \%$ of respondents preferred induction after 37 gestational weeks in 10 [24]. This result was similar to that of our survey.

Previous meta-analyses suggest that maternal hydration appeared to increase amniotic fluid volume and might be beneficial in the management of oligohydramnios $[8,25]$. In the SMFM survey, $51 \%$ of respondents thought that hydration (oral or intravenous) should be performed to gain additional amniotic fluid [9]. In the present survey, $47 \%$ of the participants believed that hydration had an effect in conservative management of $1 \mathrm{O}$. Based on this result, maternal hydration may be considered in clinical practices for 10 management. Concurrently, majority of the survey participants (87.5\%) declared that they did not consider amnioinfusion as a necessary practice in 10 management.

This study had some limitations, including a low response rate, which was probably due to a reduced number of obstetricians in the Korean Society. The low response rate might have introduced different kinds of bias to the findings. Further, this survey showed results similar to the previous KSMFM survey of 2013 regarding the preferred induction time [24]. However, we believe that several additional questions including perception of hydration and perinatal outcomes are worth investigating than the previous study to understand additional clinical practice patterns. Additionally, $64 \%$ and $83 \%$ of participants worked for tertiary hospitals in this study and the previous study, respectively [24]. This suggests that this study represented the general practices in Korea. In conclusion, KSMFM members preferred inducing labor at late-preterm to early-term, to decrease perinatal morbidity in cases of 10 , in accordance with the recent ACOG recommendation, although it is still uncertain whether labor induction improves the outcomes. Future prospective studies are needed regarding $\mathrm{IO}$ management.

\section{Acknowledgments}

This research was supported by the Basic Science Research Program through the National Research Foundation of Korea (NRF) funded by the Ministry of Science, ICT \& Future Planning (NRF-2020R1A2C4002477).

\section{Conflict of interest}

No potential conflict of interest relevant to this article was reported.

\section{Ethical approval}

The study was approved by the Institutional Review Board of Konkuk University Hospital (IRB No.2020-07-028) and performed in accordance with the principles of the Declaration of Helsinki.

\section{Patient consent}

Written informed consents were obtained. 


\section{Obstetrics \& Gynecology Science}

Vol. 63, No. 5, 2020

\section{References}

1. Dubil EA, Magann EF. Amniotic fluid as a vital sign for fetal wellbeing. Australas J Ultrasound Med 2013;16:6270.

2. Timor-Tritsch IE, Farine D, Rosen MG. A close look at early embryonic development with the high-frequency transvaginal transducer. Am J Obstet Gynecol 1988;159:676-81.

3. Chauhan SP, Cowan BD, Magann EF, Roberts WE, Morrison JC, Martin JN Jr. Intrapartum amniotic fluid index. A poor diagnostic test for adverse perinatal outcome. J Reprod Med 1996;41:860-6.

4. Chauhan SP, Sanderson M, Hendrix NW, Magann EF, Devoe LD. Perinatal outcome and amniotic fluid index in the antepartum and intrapartum periods: a metaanalysis. Am J Obstet Gynecol 1999;181:1473-8.

5. Rossi AC, Prefumo F. Perinatal outcomes of isolated oligohydramnios at term and post-term pregnancy: a systematic review of literature with meta-analysis. Eur J Obstet Gynecol Reprod Biol 2013;169:149-54.

6. Zhang J, Troendle J, Meikle S, Klebanoff MA, Rayburn WF. Isolated oligohydramnios is not associated with adverse perinatal outcomes. BJOG 2004;111:220-5.

7. Rabie N, Magann E, Steelman S, Ounpraseuth S. Oligohydramnios in complicated and uncomplicated pregnancy: a systematic review and meta-analysis. Ultrasound Obstet Gynecol 2017;49:442-9.

8. Gizzo S, Noventa M, Vitagliano A, Dall'Asta A, D'Antona $D$, Aldrich $C J$, et al. An update on maternal hydration strategies for amniotic fluid improvement in isolated oligohydramnios and normohydramnios: evidence from a systematic review of literature and meta-analysis. PLoS One 2015;10:e0144334.

9. Schwartz N, Sweeting R, Young BK, Schwartz N, Sweeting $R$, Young BK. Practice patterns in the management of isolated oligohydramnios: a survey of perinatologists. J Matern Fetal Neonatal Med 2009;22:357-61.

10. Shrem G, Nagawkar SS, Hallak M, Walfisch A. Isolated oligohydramnios at term as an indication for labor induction: a systematic review and meta-analysis. Fetal Diagn Ther 2016;40:161-73.

11. Peipert JF, Donnenfeld AE. Oligohydramnios: a review. Obstet Gynecol Surv 1991;46:325-39.

12. Munn MB. Management of oligohydramnios in preg- nancy. Obstet Gynecol Clin North Am 2011;38:387-95.

13. Mayer A, Erez O, Novack L, Bashiri A, Wiznitzer A, Mazor M. Chronic hypertension is an independent risk factor for preeclampsia and preterm delivery in women with rheumatologic diseases: a population-based study. Eur J Obstet Gynecol Reprod Biol 2007;133:157-63.

14. Brzezinski-Sinai NA, Stavsky M, Rafaeli-Yehudai $T$, Yitshak-Sade M, Brzezinski-Sinai I, Imterat $\mathrm{M}$, et al. Induction of labor in cases of late preterm isolated oligohydramnios: is it justified? J Matern Fetal Neonatal Med 2019;32:2271-9.

15. Charles D, Jacoby HE. Preliminary data on the use of sodium aminohippurate to determine amniotic fluid volumes. Am J Obstet Gynecol 1966;95:266-9.

16. Rosati P, Guariglia L, Cavaliere AF, Ciliberti P, Buongiorno $S$, Ciardulli A, et al. A comparison between amniotic fluid index and the single deepest vertical pocket technique in predicting adverse outcome in prolonged pregnancy. J Prenat Med 2015;9:12-5.

17. Magann EF, Nolan TE, Hess LW, Martin RW, Whitworth NS, Morrison JC. Measurement of amniotic fluid volume: accuracy of ultrasonography techniques. Am J Obstet Gynecol 1992;167:1533-7.

18. Rutherford SE, Smith CV, Phelan JP, Kawakami K, Ahn MO. Four-quadrant assessment of amniotic fluid volume. Interobserver and intraobserver variation. J Reprod Med 1987;32:587-9.

19. Nabhan AF, Abdelmoula YA. Amniotic fluid index versus single deepest vertical pocket: a meta-analysis of randomized controlled trials. Int J Gynaecol Obstet 2009;104:184-8.

20. Ashwal E, Hiersch L, Melamed N, Aviram A, Wiznitzer A, Yogev $Y$. The association between isolated oligohydramnios at term and pregnancy outcome. Arch Gynecol Obstet 2014;290:875-81.

21. ACOG Committee opinion No. 764 summary: medically indicated late-preterm and early-term deliveries. Obstet Gynecol 2019;133:400-3.

22. Moore TR. The role of amniotic fluid assessment in indicated preterm delivery. Semin Perinatol 2011;35:286-91.

23. Jo JH, Choi YH, Wie JH, Ko HS, Park IY, Shin JC. Fetal Doppler to predict cesarean delivery for non-reassuring fetal status in the severe small-for-gestational-age fetuses of late preterm and term. Obstet Gynecol Sci 2018;61:202-8. 


\section{Obstetrics \& Gynecology Science}

Seung Woo Yang, et al. Management of isolated oligohydramnios

24. Oh SY. Management of isolated oligohydramnios between $34+0$ and $36+6$ weeks of gestation. Korean J Perinatol 2013;24:229-36.

25. Hofmeyr GJ, Gülmezoglu AM, Novikova N. Maternal hy- dration for increasing amniotic fluid volume in oligohydramnios and normal amniotic fluid volume. Cochrane Database Syst Rev 2002;(1):CD000134. 